\title{
Access Strategies for Endovascular Intervention in the Carotid Artery: Does the Direct Cervical Approach Have A Role?
}

\author{
FRANK J. CRIADO, M.D., ERIC P. WILSON, M.D., FAISEL M. ZAMAN, \\ ROBERT J. FALCONER, M.D., and DARYOUSH SABET-PAYMAN
}

\begin{abstract}
Endovascular stent placement is rapidly evolving as a potential alternative to surgical endarterectomy in the treatment of carotid artery disease. Currently available information is preliminary and somewhat incomplete, but it does suggest that carotid catheter intervention is likely to become a frequent and successful procedure in the future. Access considerations are of key importance, with the transfemoral technique emerging as the best strategy in most instances. Direct puncture and cannulation of the cervical carotid artery is technically feasible but seldom required. When necessary, a cutdown open approach through a short incision at the base of the neck may offer advantages over the percutaneous technique. Vascular surgeons should be urged to become proficient with all forms of catheter-based procedures, and pay particular attention to carotid artery intervention.
\end{abstract}

Keywords Carotid artery, stent placement, endovascular

Prospective randomized clinical trials have shown conclusively that carotid endarterectomy (CEA) is an effective, safe, and durable treatment for highgrade stenotic lesions of the carotid bifurcation and internal carotid artery (ICA) in both symptomatic and asymptomatic patients. ${ }^{1,2}$ In fact, CEA may well be the most validated operation in contemporary vascular surgery. Given this reality, it is somewhat intriguing to witness the rapidly growing enthusiasm for endovascular intervention to treat carotid artery disease. ${ }^{3-5}$ The drive to perform catheter-based carotid procedures (instead of endarterectomy) has been fueled in large part by the aggressive attitude of cardiologists and a few interventional radiologists and vascular surgeons who are now proposing to treat carotid artery lesions nonsurgically with balloon angioplasty and endovascular stent placement. ${ }^{5-7}$

F.J.C., Director, Center for Vascular Intervention; Chief, Division of Vascular Surgery, Union Memorial Hospital/MedStar Health, Baltimore, MD; E.P.W., Associate Director, Center for Vascular Intervention, Union Memorial Hospital/MedStar Health, Baltimore, MD; F.M.Z., Medical Student, Union Memorial Hospital/MedStar Health, Baltimore, MD; R.J.F., Surgical Resident, Union Memorial Hospital/MedStar Health, Baltimore, MD; D.S-P., Medical Student, Union Memorial Hospital/MedStar Health, Baltimore, MD.

Copyright (c) 2000 by Thieme Medical Publishers, Inc., 333 Seventh Avenue, New York, NY 10001, USA. Tel. +1 (212) 584-4662. 0894-8046,p;2000,13,1,23,38,ftx,en;pvs000080 
Appropriately, carotid artery stenting continues to be considered an investigational and largely unproven therapy for carotid artery stenosis. Until more is known about efficacy and durability, a majority of interventionists today would prefer to restrict use of this new therapeutic modality to well selected patients who are less than ideal candidates for operation. There are three situations in which endarterectomy may be excessively difficult, technically unfeasible, or likely to be accompanied by increased morbidity. They all share in common adverse local anatomy: re-stenotic lesions after previous CEA, distal or very distal lesions in the high cervical or intracranial ICA, and "hostile neck" cases caused by previous radical cancer surgery and/or radiotherapy. Additionally, patients with serious medical co-morbidities that increase the risk of postoperative complications and mortality may also be considered in the same "high-risk category" that constitutes the best indication for an alternative nonsurgical approach to carotid artery disease at present. Notwithstanding, it is important to note that a majority of patients continue to be treated by CEA, which is the most frequently performed operation on most vascular surgery services.

Since July 1, 1994, we have selectively used carotid artery stenting for highrisk patients (as defined above) presenting with $70 \%$ or greater symptomatic and asymptomatic ICA lesions. ${ }^{8}$ The majority of these interventions were performed using direct cervical access through the end of 1997. Recently, we adopted the transfemoral technique which rapidly became the approach of choice in our practice. The direct cervical approach is now reserved for those infrequent instances where vascular anatomy and/or disease preclude safe catheterization of the aortic arch branches from the femoral artery.

\section{TECHNICAL PRINCIPLES OF ENDOVASCULAR ACCESS FOR CAROTID ARTERY INTERVENTION}

\section{Transfemoral Approach}

The vast majority of carotid catheter-based interventions are performed via femoral artery access. The critical technical steps include:

(1) Percutaneous catheterization by retrograde needle puncture of the common femoral artery (CFA), with placement of a $5 \mathrm{~F}$ sheath over a guidewire.

(2) Placement of a diagnostic $5 \mathrm{~F}$ curved pigtail catheter that is advanced (over the wire) to the proximal portion of the aortic arch (Fig. 1A). Needless to say, all endovascular maneuvers are monitored and guided by high-resolution C-arm fluoroscopy-digital subtraction and roadmappinig capabilities are highly desirable.

(3) Power-injector angiography of the aortic arch is obtained (in the left anterior oblique projection) to display the anatomy of the origin and 
A
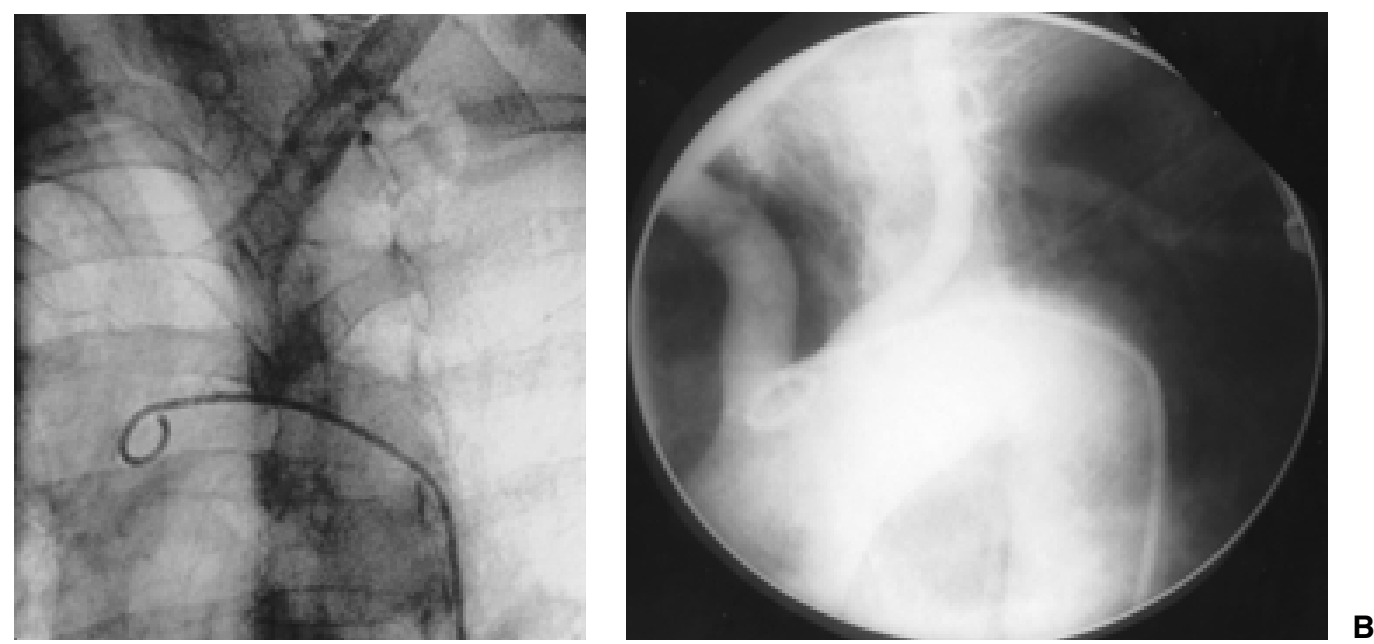

Fig. 1 (A) Placement of curved 5F pigtail catheter in the aortic arch. (B) Detailed angiographic visualization of aortic arch branches in LAO projection allows best choice of catheter and technique for selective catheterization.

proximal segments of the innominate, right and left common carotid arteries (Fig. 1B).

(4) Selective catheterization of the target aortic arch branch (i.e., innominate or left common carotid artery). This requires use of a curved (preshaped) $5 \mathrm{~F}$ catheter that is appropriately chosen for the anatomy at hand. Examples of commonly used catheters include the JB1-2 ${ }^{\circledR}$, Vitek ${ }^{\circledR}$ (Cook, Inc., Bloomington, IN), and Simmons ${ }^{\circledR}$ configurations. The JBl catheter is our most frequent choice. Engagement of the target artery is followed by passage of a 0.035 inch guidewire (Storq, Wholey, or Glidewire type) (Fig. 2). The catheter is then advanced into the proximal portion of the vessel tracking over the guidewire. Extreme caution must be exercised to prevent excessive distal advancement of the wire or catheter; they must be kept proximal to the carotid bifurcation at this point (Fig. 3).

Access difficulties (and potential complications) are not unusual during this phase of the intervention. Technical problems can be anticipated when the aortic arch is "uncoiled" or elongated in such a way that, on AP view, the apex of the arch is at a higher level than the target vessel origin (i.e., the innominate artery). Additionally, extensive calcific atherosclerosis of the aortic arch may impose significant technical challenges as well as increase the likelihood of embolic complications. As a general rule of safety, one should not persist beyond two or three catheter choices and a reasonable amount of time (20 minutes) when attempting selective catheterization of the target vessel proves difficult. Stubborn manipulations and forced maneuvers can result in vascular complications and cerebral embolization. 

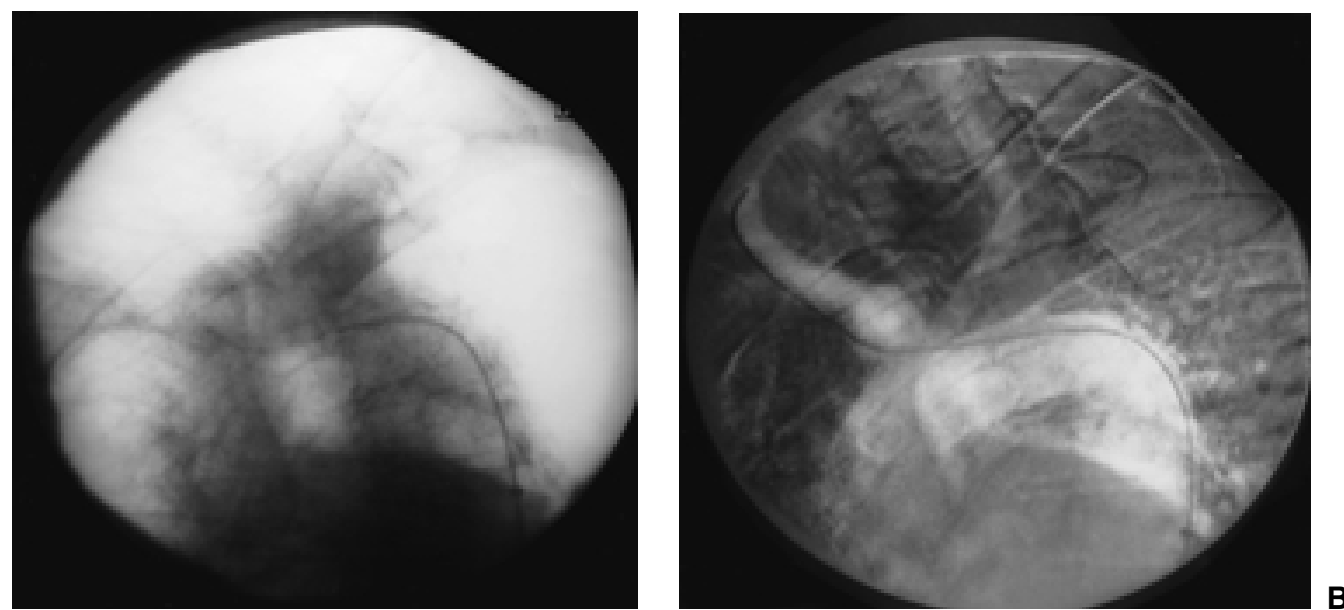

Fig. 2 (A) Use of JB-1 catheter to select innominate artery, (B) with advancement of guidewire (0.035 inch Storq) into common carotid.

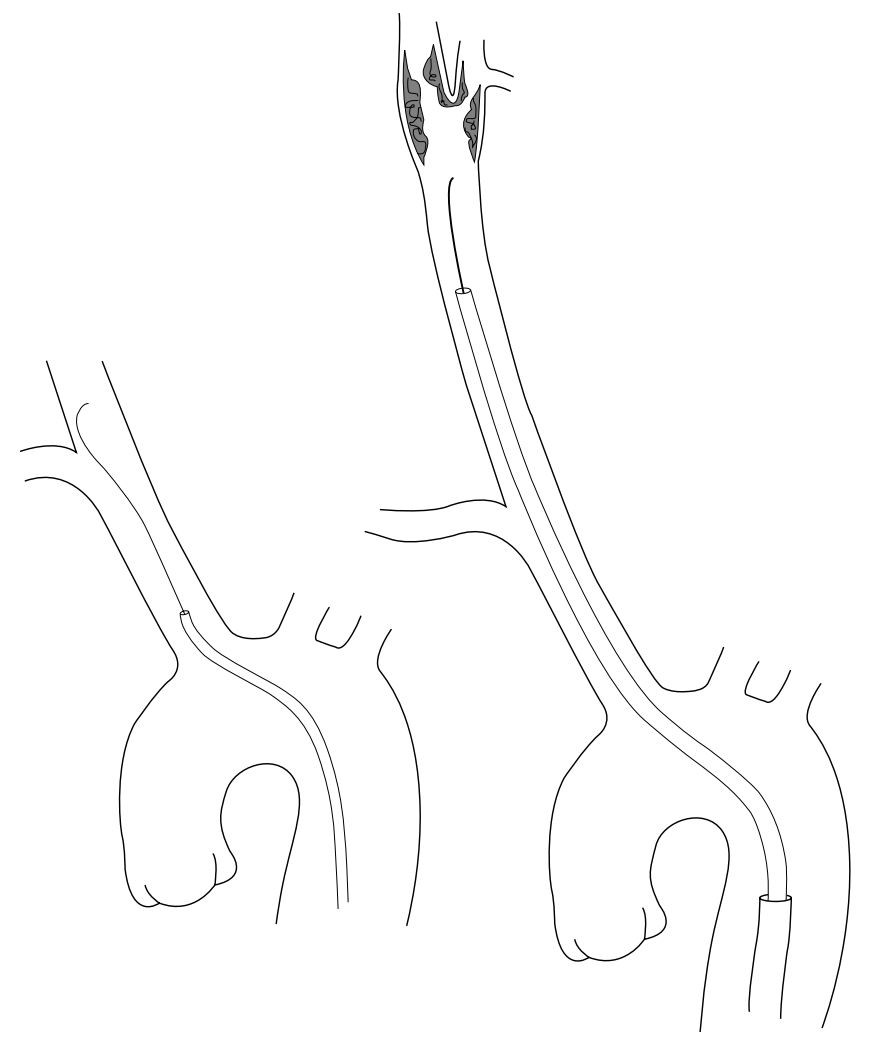

Fig. 3 Illustration of selective catheterization technique using $5 \mathrm{~F}$ JB-1 catheter (or similar) and 0.035 inch guidewire. 
A
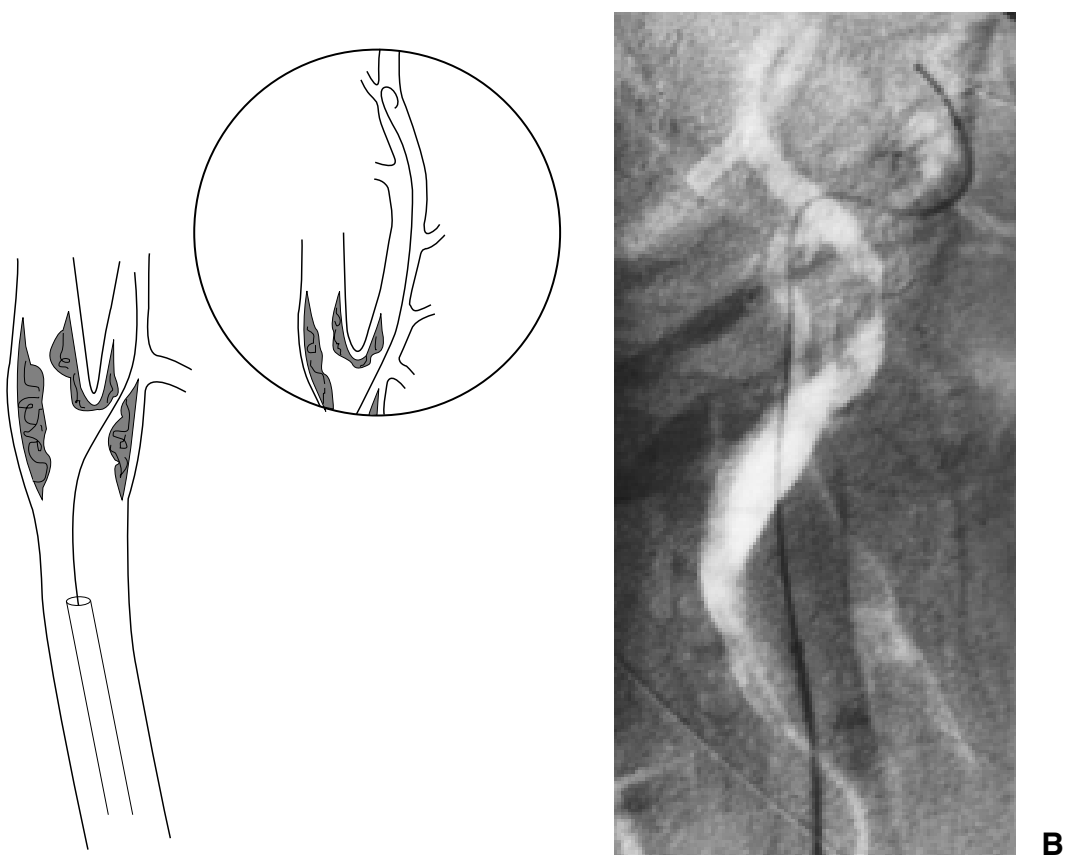

Fig. 4 (A) Initial (limited) angiographic delineation of carotid bifurcation anatomy, (B) with placement of guidewire deep into the ECA and branches.

(5) This step consists of the administration of a small amount of contrast through the catheter (which is now in the lumen of the target brachiocephalic vessel) to define the anatomy of the carotid bifurcation, and to identify the external carotid artery (ECA). The 0.035 inch guidewire can now be advanced into the ECA with deep placement in one of its branches (Fig. 4). This will provide enough wire support across the aortic arch and brachiocephalic vessels to facilitate insertion of the large interventional sheath.

(6) A 7 or 8F Shuttle Flexor sheath (Cook Inc., Bloomington, IN) is placed percutaneously (from the groin) in exchange for the initially placed $5 \mathrm{~F}$ introducer. The sheath-dilator track over the stiff (or superstiff) wire into the target common carotid artery (CCA); the abovedescribed placement of a guidewire deep into a branch of the ECA provides a firm "railroad" for this maneuver. The sheath is advanced to the distal portion of the common carotid artery, but it should never cross the carotid bifurcation (Fig. 5).

An alternative and possibly simpler technique involves placement of the long interventional sheath (to the level of the proximal descending thoracic aorta) prior to cannulating the aortic arch branch of interest. Through the sheath, a $120 \mathrm{~cm}$ or longer $5 \mathrm{~F}$ selective catheter (special order) is used for catheterization of the target aortic arch 
Fig. 5 Over-the-wire advancement of $7 \mathrm{~F}$ ( or $8 \mathrm{~F}$ ) transfemoral interventional sheath into common carotid artery. Note that dilator and sheath are not allowed to cross bifurcation.

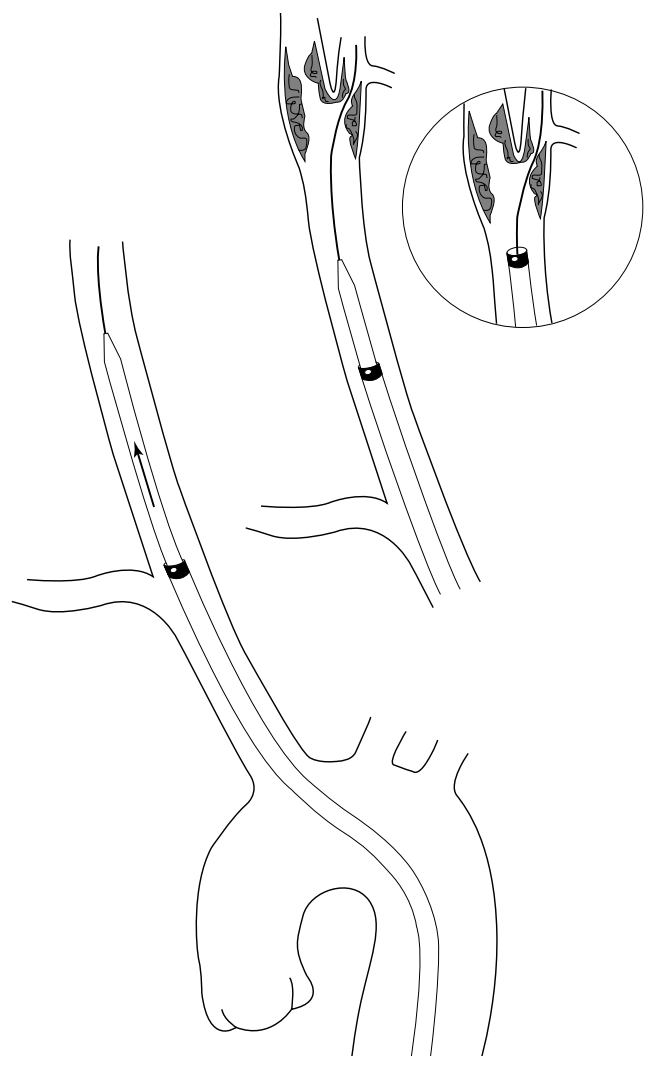

branch. Subsequent steps are similar to those described in steps 4 and 5 . The sheath can now be advanced into the CCA over the $5 \mathrm{~F}$ catheter (instead of the dilator) (Fig. 6); this technique has worked quite well in the hands of the senior author (FJC). However, it must be understood that difficulties with advancement of the sheath over the catheter (across the origin of the innominate or left CCA) should dictate a return to the fundamental steps described above; that is, removal of the catheter, and placement of the dilator in the sheath to improve trackability and facilitate forward advancement. The interventional sheath should not be forced into an aortic arch branch when it does not advance relatively easily with push-pull catheter/wire maneuvers. Aggressive attempts at sheath introduction can cause serious complications, such as, vessel dissection and atheroembolization.

(7) At this point, the 0.035 inch guidewire is removed, and a selective diagnostic angiogram obtained in the appropriate projection (oblique or lateral) to delineate the anatomy and target lesion in the carotid bifurcation and ICA (Fig. 7). More than one projection may be necessary for best anatomical definition. The ICA lesion is crossed with a 0.018 inch guidewire; we prefer to use the Roadrunner ${ }^{\circledR}$ (Cook Inc.) guidewire because it has an easily visible radiopaque distal segment that 


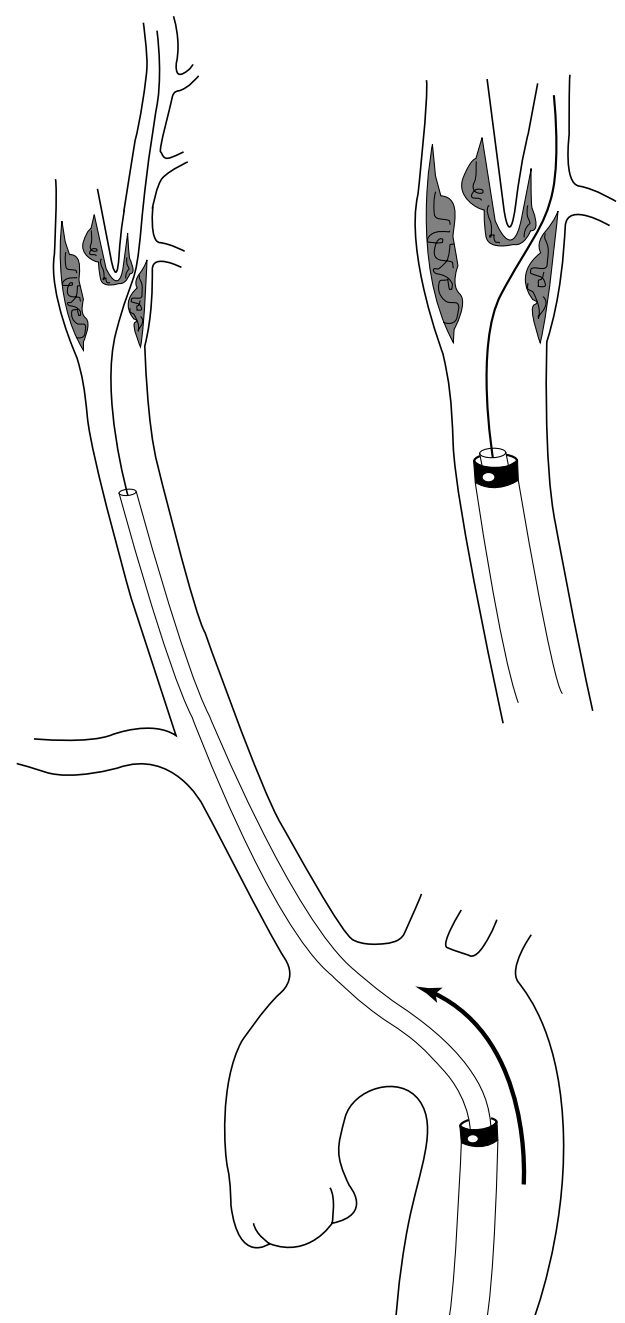

Fig. 6 Alternative technique with initial placement of interventional sheath to the proximal descending thoracic aorta. Catheterization of aortic arch branch requires use of special-order $120 \mathrm{~cm}$ or longer selective $5 \mathrm{~F}$ catheters. Sheath is advanced into distal common carotid tracking over the $5 \mathrm{~F}$ catheter/guidewire.

facilitates fluoroscopic monitoring to prevent deep intracranial penetration. The latter constitutes a critical aspect of carotid artery intervention. It must be noted that the guidewire should not be advanced through the stenosis until all preparations have been made for prompt balloon angioplasty and stent placement. Expediency and avoidance of unnecessary trauma to the lesion are of the utmost importance.

(8) Adequate systemic angicoagulation with heparin is felt to be important. We use monitoring of activated clotting time (ACT) with a target of 300 seconds. This level of anticoagulation is achieved before initiating catheter/guidewire maneuvers in the carotid artery, and maintained during the remainder of the intervention.

Although a detailed description of the techniques of balloon angioplasty and stent placement is beyond the scope of this article, it is appropriate to include the underlying technical principles. Preliminary balloon dilation of 
Fig. 7 Selective pre-stenting angiogram of left carotid artery on patient with "hostile neck" and a critical ICA lesion. Note that the large transfemoral interventional sheath has been placed to the mid CCA.

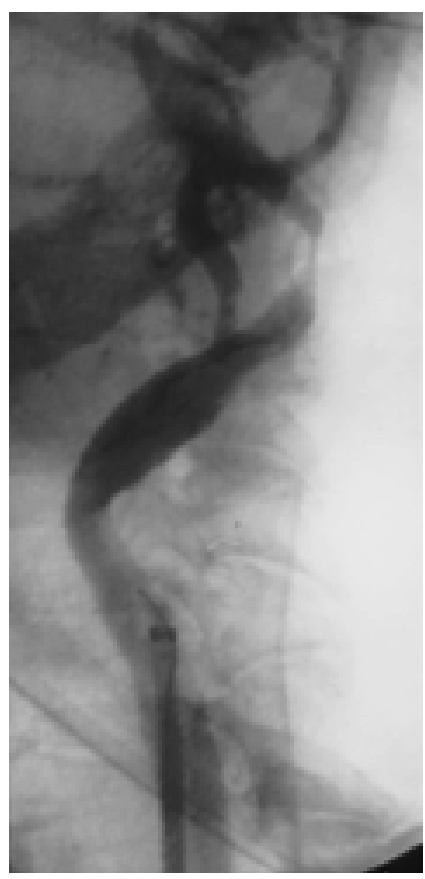

the lesion is felt to be important. We use a $4 \mathrm{x} 40 \mathrm{~mm}$ low-profile (or smallvessel) balloon angioplasty catheter for this purpose. Our current stent of choice is the self-expanding nitinol SMART ${ }^{\circledR}$ stent (Cordis Endovascular, Warren, NJ); the 7 to $8 \mathrm{~mm}$ (diameter) X $40 \mathrm{~mm}$ (length) sizes suit most situations quite well. A recent modification in our technique (with the SMART $^{\circledR}$ device) has been the omission of post-balloon dilatation after stent placement; it is probably unnecessary, and adds risks of cerebral embolization. A nitinol stent that is oversized by 1 to $2 \mathrm{~mm}$ beyond the estimated normal diameter of the ICA uniformly results in excellent angiographic contour, especially when assessed 5 to 10 minutes after initial deployment. Minor luminal defects and $<20 \%$ residual stenosis are acceptable anatomic outcomes in carotid artery intervention (Fig. 8); the pursuit of a "perfect" angiographic appearance implies the frequent need for further balloon expansion that is likely to increase the danger of embolic complications.

\section{Direct Cervical Approach}

Percutaneous antegrade puncture of the cervical CCA is not difficult. Instead of the "standard" $18 \mathrm{~g}$ arterial needle, a $21 \mathrm{~g}$ micropuncture set (Cook) allows atraumatic entry that is likely to cause less pain and with lower risk of nerve injury or hematoma formation. The puncture should be made as proximal (low) as possible to have enough intraluminal distance for placement of the sheath without crossing the bifurcation. Preprocedure localization of the bifurcation by duplex ultrasound enables precise skin 


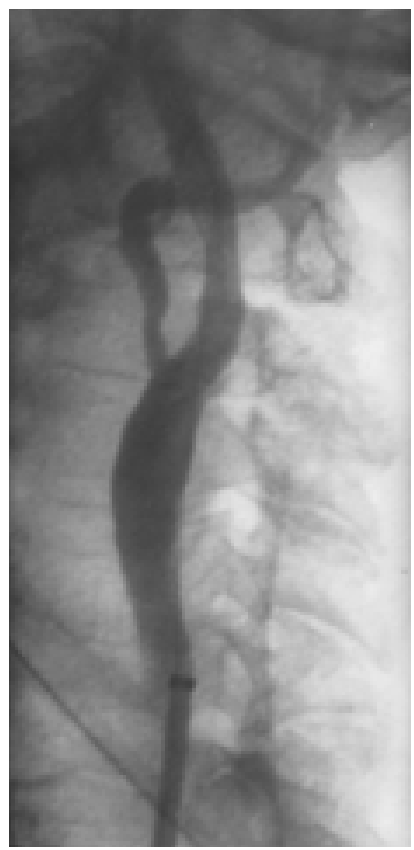

Fig. 8 Same patient after placement of SMART stent in left ICA. Lesion was predilated with $3.5 \mathrm{~mm}$ smallvessel PTA balloon, but postdilatation was omitted. Minimal residual stenosis present.

mapping to guide proper selection of the best puncture site (Fig. 9). ${ }^{8}$ On occasion, a very low-lying bifurcation may preclude direct cervical access for ICA intervention.

While obtaining percutaneous access offers, little if any, difficulty, achieving secure hemostasis upon sheath removal is quite another story. External

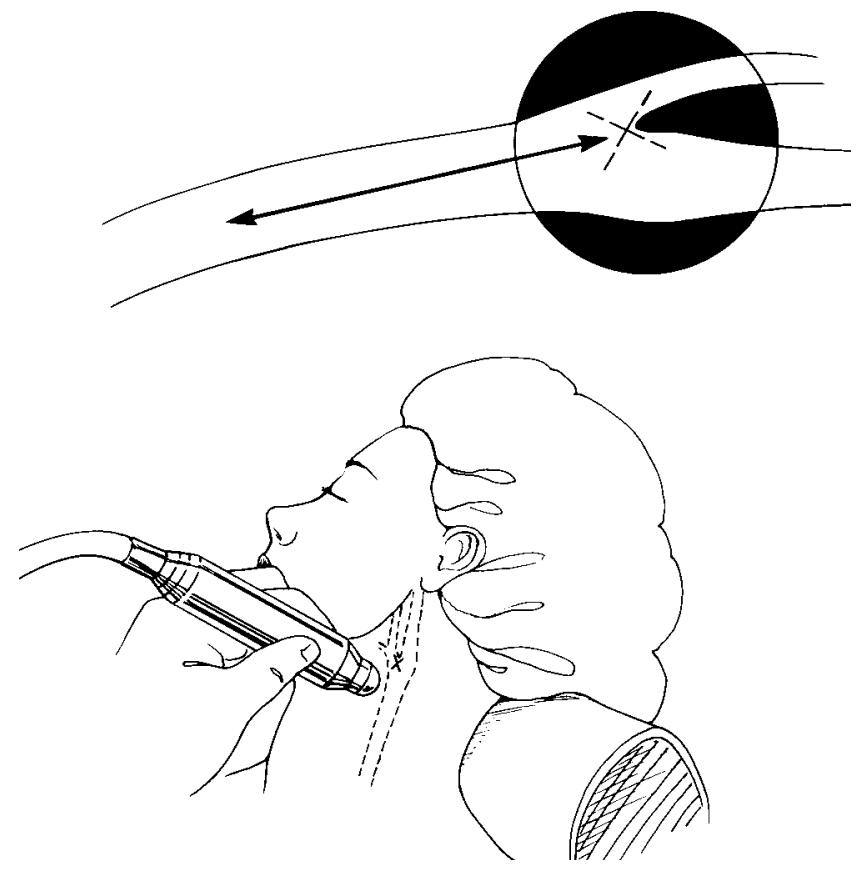

Fig. 9 Duplex ultrasound mapping and external skin marking of carotid bifurcation facilitates direct cervical puncture, and reveals whether there is sufficient distance proximal to the bifurcation to accommodate introducer sheath. 

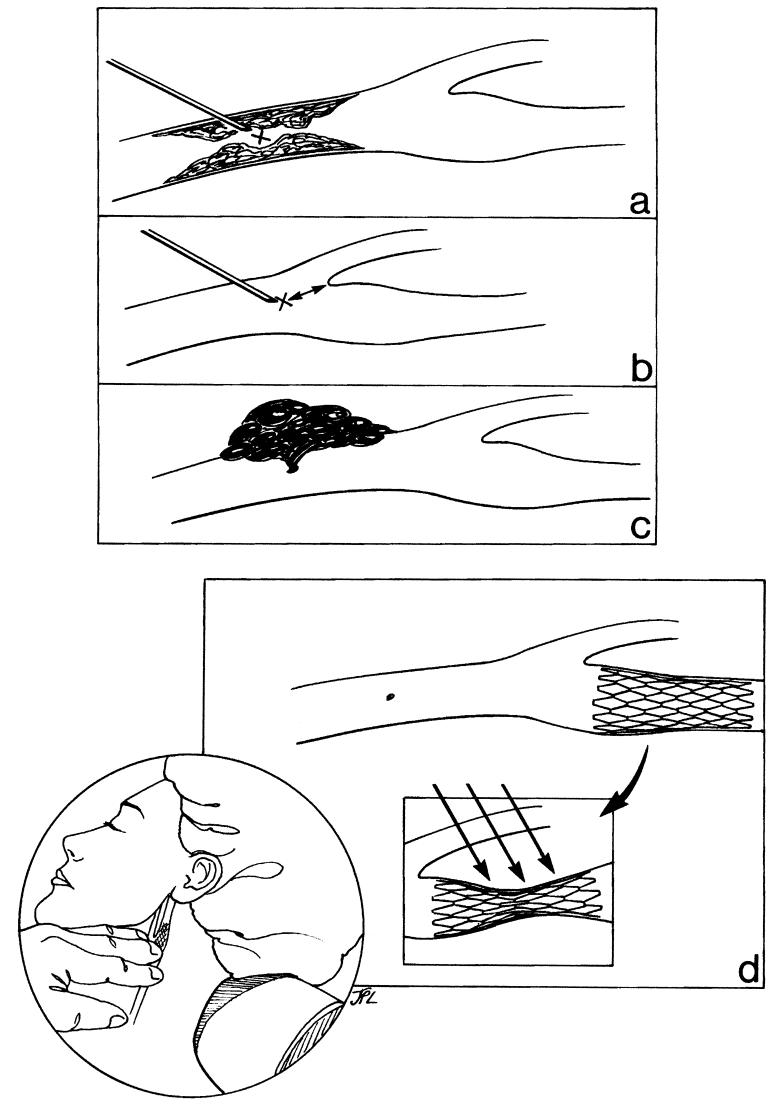

Fig. 10 (A) Illustration of potential disadvantages of direct cervical puncture, including presence of atheromatous disease in the common carotid. (B) Low-lying bifurcation with insufficient length for proximal sheath placement. (C) Ineffective compression leading to hematoma formation or hemorrhage. (D) External compression of puncture site can also lead to deformation or crushing of a balloon-expandable stent.

compression can be difficult and ineffective, and very uncomfortable if the patient is conscious (Fig. 10). This has led to the adoption of the surgical cutdown approach with suture-repair of the arterial puncture site. ${ }^{8,9}$ The technique is as follows:

(1) A 3 to $5 \mathrm{~cm}$ low-neck longitudinal incision is made along the anterior border of the sternal head of the sternocleidomastoid muscle. Exposure of proximal CCA requires deep lateral dissection under the muscle. Local anesthesia with intravenous sedation or general endotracheal anesthesia are appropriate choices, with the latter being the preferred strategy whenever possible. 


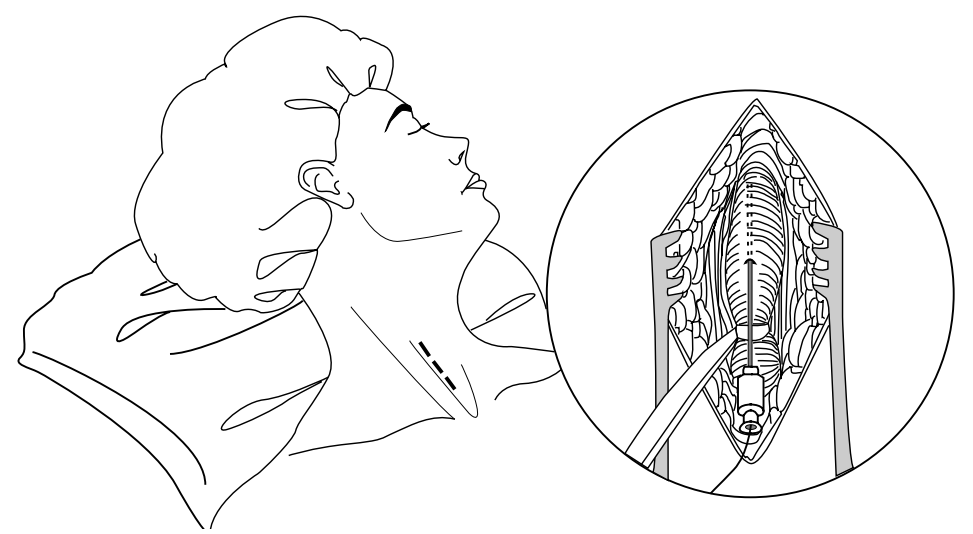

Fig. 11 Short-incision surgical cutdown for exposure of proximal CCA, and direct access utilizing the micropuncture $2 \lg$ set.

(2) The CCA is controlled circumferentially with a single Silastic vessel loop.

(3) Direct needle puncture of the artery and introduction of guidewire are straightforward (Fig. 11). A small 5F catheter (from the micropuncture set) is placed intraluminally over the wire, and a small "puff" of radiocontrast is administered to image the carotid bifurcation and identify the ECA. Oblique or lateral projections may better display the anatomy of the bifurcation. Once the ECA has been identified, the 0.035 inch guidewire is advanced deep into one of its branches (Fig. 12A,B). Care must be exercised not to enter the ICA at this time.

(4) A 25 to $35 \mathrm{~cm}$ long 7F sheath is now placed into the artery (over the guidewire) but allowing only 2 to $4 \mathrm{~cm}$ intraluminal advancement to keep it from reaching the bifurcation and ICA (Fig. 12C,D). The long sheath "moves the site of action" down to the lower chest or upper abdominal areas, well removed from the neck and image intensifier of the C-arm fluoroscope. This facilitates all subsequent steps, and mirrors the remote-access approach that constitutes the hallmark of endovascular intervention everywhere.

(5) Once the 7F sheath has been placed to the level of the distal CCA, a detailed selective carotid angiogram is obtained (Fig. 12E). The intervention can now proceed in much the same manner as that described for the transfemoral approach, including lesion crossing with a 0.018 inch guidewire (Fig. 12F), balloon pre-dilatation, and stent deployment.

(6) Upon completion, the sheath is removed, and the arterial puncture site repaired with one or two simple vascular sutures to achieve secure hemostasis (without causing vessel stenosis). 


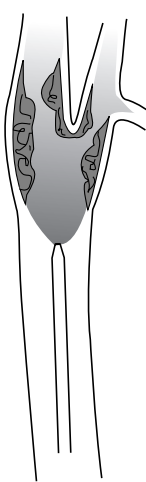

A

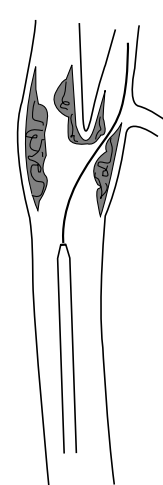

B

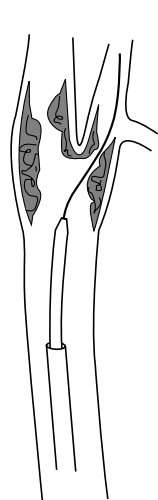

C

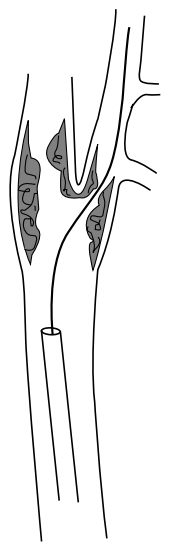

D

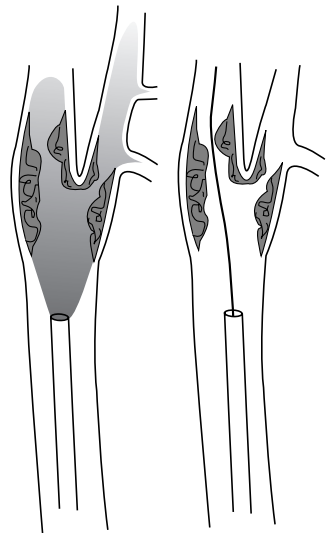

$\mathbf{F}$

Fig. $12(A, B)$ Initial angiographic visualization of carotid bifurcation allows placement of 0.035 guidewire into ECA. (C,D) Placement of 7 or $8 \mathrm{~F}$ introducer sheath into CCA. (E,F) Followed by detailed angiographic visualization to guide balloon dilatation and stent placement.

\section{SUMMARY OF CLINICAL EXPERIENCE AND RESULTS}

Carotid artery stenting has been performed on selected patients in our practice since 1994. The direct cervical approach was used almost routinely in the initial series of 52 procedures. ${ }^{8}$ Technical strategies evolved with time and growing experience; while percutaneous antegrade puncture of the cervical common carotid artery was found to be relatively simple to perform, obtaining secure hemostasis upon removal of the introducer sheath was often a challenge. The open approach though a short cutdown incision at the base of the neck was adopted to address this dilemma as surgical exposure enabled reliable hemostasis through suture-repair of the puncture site.

Adoption of the (remote) transfemoral access technique (December 1997) represented a logical evolution as we recognized the unequivocal superiority of this approach for the vast majority of patients. It has been used in 34 of the last 36 carotid interventional procedures. The cervical approach became necessary in only $2(5 \%)$ of these cases because of unsuitable anatomy and/or disease precluding safe transfemoral catheterization.

In our overall experience (July 1, 1994 through October 31, 1999) with 88 carotid endovascular interventions (instead of CEA), 60 patients $(70 \%)$ were selected for stenting because they presented with one or more local anatomi$\mathrm{cal} /$ lesion factors felt to increase the risk of CEA or-occasionally-make it technically unfeasible (Table 1 ). The remaining 28 patients $(30 \%)$ undergoing stent placement did not have any obvious contraindications to CEA.

Periprocedural complications are summarized in Table 2. Of the two transient ischemic attacks (TIA) instances, one was likely access-related following 
Table 1 Carotid Artery Stenting N 88

\begin{tabular}{ll}
\hline Indications & \\
\hline ICA Lesions & 31 \\
Restenosis & 16 \\
"Hostile Neck" & 13 \\
None ("by choice") & 28 \\
\hline
\end{tabular}

stubborn attempts at transfemoral catheterization of the left common carotid artery in the face of unfavorable aortic arch anatomy and signficant calcific atherosclerotic disease. The other three neurologic events were probably the result of balloon/stent-induced embolization. Cerebral protection devices were not used during this experience.

\section{COMPARATIVE ASSESSMENT OF THE TWO APPROACHES- WHEN TO USE CERVICAL ACCESS}

It is unquestionable that most carotid interventions are and will be performed transfemorally. The technique is a must-know for all physicians planning to undertake these procedures. Selective arch-vessel catheterization with placement of the transfemoral sheath (or guiding catheter) is an advancedlevel intervention to be tried only by seasoned endovascular specialists, and after appropriate training and proctored initial experience. Technical difficulties are not uncommon; successful troubleshooting requires ample knowledge of selective catheters, proper guidewire choices, and imaging strategy. But additionally, there are (perhaps) 5 to $10 \%$ of instances where anatomy and/or disease of the aortic arch and its branches (or aortoiliac arteries) pose serious interventional challenges (Fig. 13) that may on occasion preclude successful transfemoral catheterization of the carotid arteries. The direct cervical approach offers an alternative access pathway for such patients. This is particularly attractive in case of restenosis after previous CEA, and distal ICA lesions that may not be suitable for a surgical approach, but is less appealing

Table 2 Carotid Artery Stenting N 88

\begin{tabular}{lr}
\hline Complications (30 Days) \\
\hline Stroke & 2 \\
TIA & 2 \\
Death & 0 \\
& \\
\hline
\end{tabular}




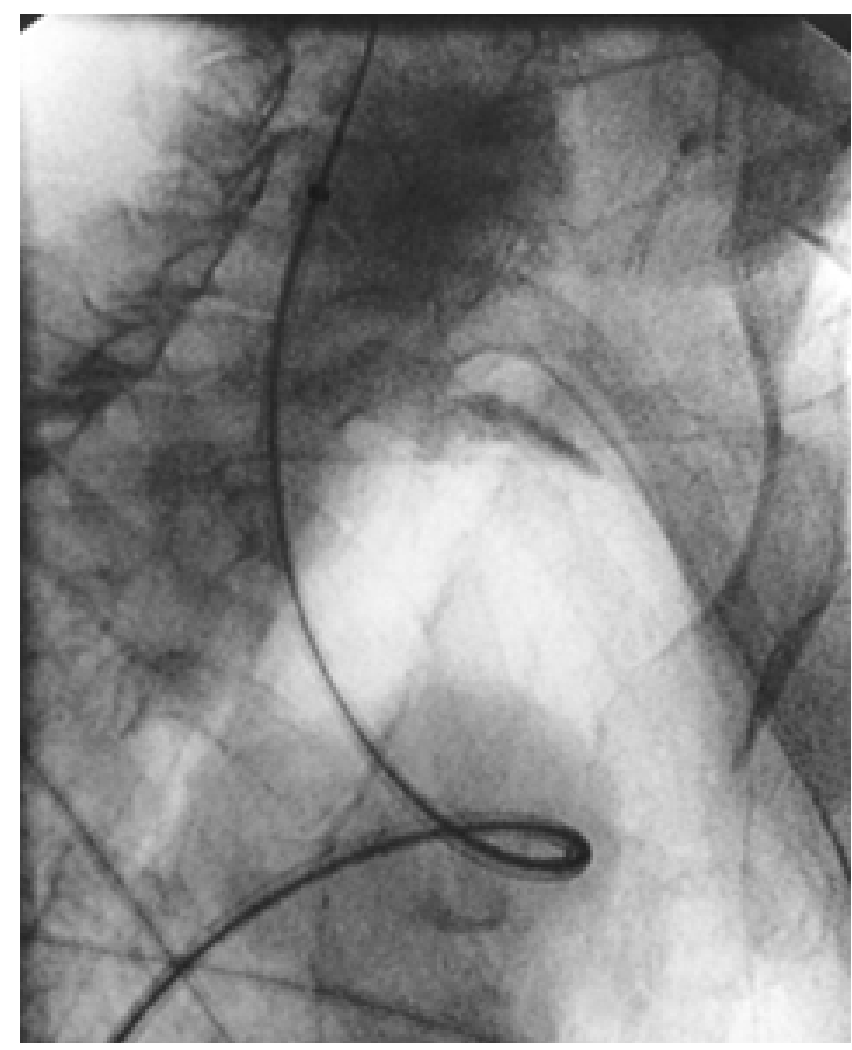

Fig. $137 \mathrm{~F}$ interventional sheath has been placed in the right CCA, going across a severely tortuous, "uncoiled" aortic arch. A Wallstent was successfully deployed in the right ICA/CCA in this patient.

on patients with "hostile necks" after previous radical surgery and permanent tracheostomy.

We advocate strongly the open cutdown approach for cervical access to prevent puncture-site related complications after sheath removal. Development of puncture-site closure devices for the cervical CCA may one day allow safe percutaneous intervention. Use of a long sheath for direct cervical access may seem odd and clumsy at first; however, it really works well by recreating the "remote access scenario" of endovascular intervention in general. Working through a short sheath is cumbersome because of practical difficulties related to the anatomical area and proximity to the image intensifier.

\section{OVERVIEW AND CONCLUSION}

The following statements represent (in our view) the key concepts and guiding principles that best define the topic of endovascular carotid procedures at present: 
- Endovascular intervention remains unproven for the treatment of carotid artery disease. Results of well-designed, randomized clinical trials are eagerly awaited to confirm (or refute) the efficacy and durability of endoluminal stent placement.

- Risk of cerebral embolization is sufficiently serious as to temper the enthusiasm of many interventionists who "can't wait" to treat most carotid artery stenoses by percutaneous stent placement.10,11 Current developments with evolving "carotid-dedicated" stent devices and emboli protection technologies may prove critically important to enhance safety and efficacy.

- The transfemoral approach is the access strategy of choice in most cases. This is not an entry-level procedure. Extensive previous endovascular experience and additional training in carotid interventional techniques are an absolute requirement for all physicians who wish to perform these techniques. ${ }^{12}$

- Direct cervical access is a valuable (albeit infrequently necessary) alternative that may be used when the transfemoral technique proves excessively difficult or impossible.

- It is the authors' conviction that endovascular intervention is likely to become an increasingly safer and widely used therapeutic modality for treatment of carotid artery disease. Vascular surgeons should watch these developments with great interest, and consider possible training pathways to acquire endovascular skills in general, and carotid interventional capabilities in particular.

\section{REFERENCES}

1. North American Symptomatic Carotid Endarterectomy Trial Collaborators. Beneficial effect of carotid endarterectomy in symptomatic patients with high-grade carotid stenosis. N Engl J Med 1991;325:445-453

2. Asymptomatic Carotid Atherosclerosis Study. Clinical advisory: carotid endarterectomy for patients with asymptomatic internal carotid artery stenosis. Stroke 1994;25: $2523-2524$

3. Beebe HG, Archie JP, Baker WH, et al. Concern about safety of carotid angioplasty. Stroke 1996;27:197-198

4. Roubin GS, Yadav S, Iyer SS, et al. Carotid stent-supported angioplasty: A neurovascular intervention to prevent stroke. Am J Cardiol 1996;3:132-139

5. Criado FJ, Wellons E, Clark NS. Evolving indications for and early results of carotid artery stenting. Am J Surg 1997;174:111-114

6. Bergeron P. Carotid angioplasty and stenting: is endovascular treatment for cerebrovascular disease justified? J Endovasc Surg 1996;3:129-131

7. Diethrich EB. Indications for carotid artery stenting: a preview of the potential derived from early clinical experience. J Endovasc Surg 1996;3:132-139

8. Criado FJ, Wellons E, Clark NS. Is the direct cervical approach a reasonable option for endovascular intervention in the carotid artery? J Invas Cardiol 1998;10:292-297

9. Diethrich EB, Marx P, Wrasper R, et al. Percutaneous techniques for endoluminal carotid interventions. J Endovas Surg 1996;3:182-202 
10. Ohki T, Marin ML, Lyon RT, et al. Ex vivo human carotid artery bifurcation stenting: correlation of lesion characteristic with embolic potential. J Vasc Surg 1998;27:463-471

11. Mathur A, Roubin GS, Iyer SS, et al. Predictors of stroke complicating carotid artery stenting. Circulation 1998;97:1239-1245

12. Wholey $\mathrm{MH}$, Wholey $\mathrm{MH}$, Bergeron $\mathrm{P}$, et al. Current global status of carotid artery stent placement. Cathet Cardiovasc Diagn 1998;44:1-6 
Criado and colleagues describe carotid angioplasty-stenting preferentially performed by the transfemoral route, but also outline a technique for percutaneous or direct carotid exploration and intervention. We have also performed the transfemoral approach in $50 / 52$ procedures; our two cases of direct operative exploration of the cervical common carotid artery (CCA) were performed in patients with severe aortoiliac occlusive disease. Criado's technical recommendations are excellent and generally have been used in our program, initiated in October, 1996 primarily for management of restenotic lesions within 3 years of carotid endarterectomy (CEA). ${ }^{1}$ Forty of our 52 cases have been treated for intimal hyperplastic restenoses, while the remainder have been higher risk NASCET-ineligible patients. While 28/88 (32\%) of his cases were performed in the current report for reasons of "choice," none of our cases was so categorized. Until data are available from clinical trials, we recommend CEA for all eligible cases.

Variations do exist in our procedural protocol. We use hand-injection angiography of the aortic arch and generally do not perform a formal arch injection via a pigtail catheter. We preferentially use the Vitek catheter as recommended by Roubin ${ }^{2}$ to cannulate the aortic arch branches. Rather than using an 0.035-inch guidewire placed into branches of the external carotid artery (ECA), we prefer to use an 0.035-inch Amplatz stiff guidewire over which the $8 \mathrm{~F}$ guide sheath has superior tracking qualities. Although we do not regard these issues as major technical differences, we offer the alternative information to demonstrate the breadth of choices available for this evolving endovascular procedure.

\section{REFERENCES}

1. Hobson RW II, Goldstein JE, Jamil Z, et al. Carotid restenosis: Operative and endovascular management. J Vasc Surg 1999;29:228-238

2. Roubin GS, Yadav S, Iyer SS, Vitek J. Carotid stent-supported angioplasty: A neurovascular intervention to prevent stroke. Am J Card 1996;783:8-12 
All of us in the endovascular field owe a debt of gratitude to Dr. Hobson, for his embrace of angioplasty/stenting in the treatment of (some) carotid artery lesions is nothing short of an endorsement of the technique by mainstream academic vascular surgery. It is the kind of support that is needed to retain vascular surgical leadership in this rapidly evolving field.

I find only a few areas of disagreement in Dr. Hobson's Expert Commentary: The criticism regarding our "choice" for a nonsurgical approach in $32 \%$ of the reported cases is well taken. However, we continue to offer stenting increasingly to patients who have no particular contraindication to surgerywhen angiographic appearance of the stenosis and access anatomy are suitable for catheter-based intervention. To date (July, 2000), we have performed the technique in an additional 55 patients without a single stroke or major complication. It seems to us that careful case selection and impeccable technical execution are the two critical factors in achieving such results. Cerebral protection devices may further enhance safety in the future.

While practically agreeing with Dr. Hobson, I would argue phylosophycally with the statement that "we recommend CEA for all eligible cases": 77\% $(40 / 52)$ of their procedures involved treatment of post-CEA restenosis which, by some accounts, ${ }^{1}$ can be managed by repeat operation with similar technical ease and safety as primary CEA. So, it would be fair to state that a "choice" exists in such patients as well. And, in fact, the majority of restenosis cases continue to be treated by redo CEA (or patch repair) at this time.

While hand-injection aortography is possible, use of a power injector is unquestionably the optimal technique to achieve angiographic imaging with the least amount of radiocontrast.

The comments on catheterization technique and access deserve a short reply. The Vitek catheter is indeed a good tool for engagement of the vessel origin in the arch, but further advancement into the artery can be quite difficult because of the very tight configuration of the catheter. We find the JB12 catheters more user-friendly, and adequate in about $85 \%$ of instances. More difficult anatomy can be managed with the SIMl or similar catheters.

Finally, the comment on access maneuvers to facilitate placement of the long interventional sheath is worthy of clarification. It seems to me the issue is not whether to use a superstiff or another good-support guidewire, but where to "anchor" the wire for this important technical step. The external carotid and its branches provide the optimal outlet, with little or no risk of manipulating the internal carotid at this point in the procedure. We (and

Copyright (C) 2000 by Thieme Medical Publishers, Inc., 333 Seventh Avenue, New York, NY 10001, USA. Tel. +1 (212) 584-4662. 0894-8046,p;2000,13,1,41,42,ftx,en;pvs000082 
most other carotid interventionists) feel strongly about using this technique whenever possible.

\section{REFERENCES}

1. Hill BB, Olcott IV C, Dalman RL, et al. Reoperation for carotid stenosis is as safe as primary carotid endarterectomy. J Vasc Surg 1999;30:26-35 